\title{
Effect of node status on breast cancer survival by subtype: a single-center retrospective cohort study
}

\author{
Weibin Lian ${ }^{1 \#}$, Fangmeng Fu ${ }^{2 \#}$, Debo Chen ${ }^{1 \#}$, Chuan Wang ${ }^{2}$ \\ ${ }^{1}$ Department of Breast Surgery, The Affiliated Quanzhou First Hospital of Fujian Medical University, Quanzhou, China; ${ }^{2}$ Department of Breast \\ Surgery, The Affiliated Union Hospital of Fujian Medical University, Fuzhou, China \\ Contributions: (I) Conception and design: W Lian, C Wang; (II) Administrative support: W Lian, C Wang, D Chen; (III) Provision of study materials \\ or patients: W Lian, C Wang, F Fu; (IV) Collection and assembly of data: W Lian, F Fu; (V) Data analysis and interpretation: W Lian, D Chen; (VI) \\ Manuscript writing: All authors; (VII) Final approval of manuscript: All authors. \\ \#These authors contributed equally to this work. \\ Correspondence to: Weibin Lian. Department of Breast Surgery, The Affiliated Quanzhou First Hospital of Fujian Medical University, No. 250 East \\ Street, Licheng District, Quanzhou 362000, China. Email: weibinlian@fjmu.edu.cn; Chuan Wang. Department of Breast Surgery, The Affiliated \\ Union Hospital of Fujian Medical University, No. 29 Xinquan Road, Gulou District, Fuzhou 350001, China. Email: chuanwang1968@Outlook.com.
}

\begin{abstract}
Background: Nodal involvement and molecular subtypes were used as independent prognostic indicators in women with breast cancer. However, they did not adequately address the effect of node status by subtype in outcomes.

Methods: We performed a retrospective review of data from 2004 to 2011 from the Affiliated Union Hospital of Fujian Medical University with newly diagnosed stage I to III breast cancer to investigate the relationship between node status and 5-year disease-free survival (DFS) and breast cancer-specific survival (BCSS) by molecular subtype. The Cox proportional hazards model was used for multivariate analysis.

Results: Median follow-up time was 6.4 years. Luminal HER2 and luminal B were the subtypes with a higher percentage of nodal involvement and high-volume nodal involvement ( $\geq 4$ positive lymph node) than luminal A. The effect of node status on the prognosis varied with molecular subtype. There was no difference in 5-year DFS and BCSS between stage N1 or N2 and N0 groups in patients with luminal A disease. Nodal involvement in women with the luminal B, luminal HER2, and triple-negative subtypes showed significant difference for 5-year DFS and BCSS compared to the node negative group.
\end{abstract}

Conclusions: Nodal involvement seems to be associated with worse survival in women with the luminal B, luminal HER2, and triple-negative subtypes, but not with the luminal A subtype.

Keywords: Breast cancer; nodal status; subtype; survival

Submitted Feb 20, 2020. Accepted for publication Aug 28, 2020.

doi: $10.21037 /$ tcr-20-1117

View this article at: http://dx.doi.org/10.21037/tcr-20-1117

\section{Introduction}

Breast cancer is the most frequent type of carcinoma and the second most common cause of death from carcinomas in women (1-3). Prognostic variables for breast cancer have traditionally included node status, histological grade, tumor size, hormone receptor (HR) status, and human epidermal growth factor receptor 2 (HER2) expression. Numerous studies have suggested that nodal involvement is the most crucial prognostic parameter, and play a pivotal role in instructing treatment $(4,5)$. In 2000, Perou et al. identified breast tumor cells that shared gene expression patterns and clustered it into four subtypes: luminal epithelial/estrogen receptor (ER) positive, normal-breast-like, basal-like, and/ or cells with over-expression of the human epidermal growth factor receptor 2 (HER2) gene (6). Previous studies reported that molecular subtypes are associated with different risks of early disease recurrence, metastases, and survival (7-9). Although nodal involvement and molecular subtypes are both independently well-recognized prognostic indicators, it remains unclear whether there is 
Table 1 Patient and tumor characteristics

\begin{tabular}{|c|c|c|}
\hline Parameters & No. of patients & Percentage (\%) \\
\hline \multicolumn{3}{|l|}{ Age, years } \\
\hline$\leq 40$ & 525 & 25.1 \\
\hline$>40$ & 1,564 & 74.9 \\
\hline \multicolumn{3}{|l|}{ Tumor size, cm } \\
\hline$\leq 2$ & 862 & 41.3 \\
\hline$>2$ & 1,227 & 58.7 \\
\hline \multicolumn{3}{|l|}{ Node status } \\
\hline NO & 1,119 & 53.6 \\
\hline N1 & 521 & 25.0 \\
\hline N2 & 256 & 12.2 \\
\hline N3 & 193 & 9.2 \\
\hline \multicolumn{3}{|l|}{ Grade } \\
\hline Low/moderate & 1,453 & 69.5 \\
\hline High & 492 & 23.6 \\
\hline Unknown & 144 & 6.9 \\
\hline \multicolumn{3}{|l|}{$\mathrm{Ki}-67$} \\
\hline Low & 763 & 36.5 \\
\hline High & 796 & 38.1 \\
\hline Unknown & 530 & 25.4 \\
\hline \multicolumn{3}{|l|}{ Subtype } \\
\hline Luminal A & 464 & 22.2 \\
\hline Luminal B & 653 & 31.3 \\
\hline Luminal HER2 & 294 & 14.1 \\
\hline HER-2 & 341 & 16.3 \\
\hline TN & 337 & 16.1 \\
\hline \multicolumn{3}{|l|}{ ER } \\
\hline Positive & 1,400 & 67.0 \\
\hline Negative & 689 & 33.0 \\
\hline \multicolumn{3}{|l|}{ PR } \\
\hline Positive & 1,284 & 61.5 \\
\hline Negative & 805 & 39.5 \\
\hline \multicolumn{3}{|l|}{ HER2 } \\
\hline Positive & 638 & 30.5 \\
\hline Negative & 1,451 & 69.5 \\
\hline
\end{tabular}

an association between these two factors $(10,11)$. He et al. have reported that triple-negative breast cancer is associated with a reduced risk of nodal involvement compared to other subtypes (12). A study by Liao et al. revealed significant differences in overall survival (OS) according to the node status in luminal A, luminal B, and luminal HER2 subtypes, and with recurrence-free survival (RFS) in the luminal $B$ and luminal HER2 subtypes after adjustments for age and tumor size (13). Thus, it can be seen that node status is associated with molecular subtypes in outcomes, and nodal involvement may not always be an independent risk factor for some molecular subtypes. To address this, we retrospectively investigated the effect of the node status on the 5-year disease-free survival (DFS) and breast cancerspecific survival (BCSS) with different molecular subtypes of breast cancer. We present the following article in accordance with the STROBE reporting checklist (available at http://dx.doi.org/10.21037/tcr-20-1117).

\section{Methods}

\section{Patients}

We retrospectively reviewed the medical records of breast cancer patients with stages I to III disease [American Joint Committee on Cancer (AJCC) staging, $7^{\text {th }}$ edition] treated from 2004 to 2011 in the Affiliated Quanzhou First Hospital of Fujian Medical University. We excluded 49 patients who had either had a previous diagnosis of malignant tumor or who were missing follow-up information. The study was conducted in accordance with the Declaration of Helsinki (as revised in 2013). The study was approved by Affiliated Union Hospital of Fujian Medical University Ethics committee (No. 002934) and informed consent was taken from all the patients. The demographics and clinicopathological information from their medical records were regrouped and are given in Table 1 .

\section{Definitions}

Node status was defined by TNM classification as proposed by the American Joint Committee on Cancer for grouping patients with respect to prognosis. Categorizing the number of metastatic lymph nodes into N0 (0), N1 $(\leq 3)$, $\mathrm{N} 2$ (4 to 9), and N3 ( $\geq 10)$ groups, and the N0 group acted as the reference group. The ER and PR expression were 


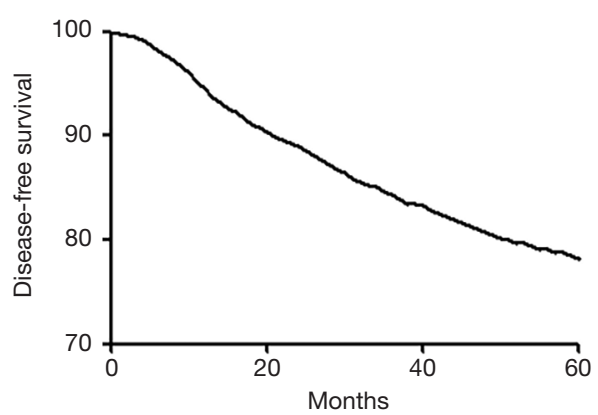

Figure 1 Kaplan-Meier curves for 5-year DFS. DFS, disease-free survival.

assessed using immunohistochemical (IHC) staining. An ER expression of more than $1 \%$ was considered positive, and expression of more than $20 \%$ for PR was classified as high expression. HER2 positivity was defined as a 3+ staining intensity score at IHC analysis for the HER2 protein or for $H E R 2$ gene amplification by fluorescence in situ hybridization (FISH) (14). According to the reported from Cheang et al. (15), we defined expression of $14 \%$ or greater as a high Ki-67 level and less than $14 \%$ as a low level of expression. If $\mathrm{Ki}-67$ results are not available, the grade can be used as a surrogate for the molecular subtypes (16). According to the St. Gallen International Breast Cancer Conference (2013 or 2015), we identified the following molecular subtypes; luminal A (ER positive and PR high, HER2 negative and Ki-67 low expression or low/intermediate grade), luminal B (ER positive and HER2 negative and either PR low or Ki-67 high or high grade), luminal HER2 (ER positive and HER2 positive), HER2 (ER negative, PR negative and HER2 positive), and triple-negative (TN; ER negative, PR negative, and HER2 negative) $(17,18)$.

\section{Statistics}

Statistical analysis was performed using SPSS version 22.0 (IBM Corporation, New York, USA). Univariate and multivariate analyses of breast cancer survival comprising the patient's age, tumor size, histologic grade, Ki-67, and molecular subtype were performed. Furthermore, we conducted Multivariable Cox proportional hazards regression with adjustments, including age, tumor size, and histologic grade, to estimate the hazard ratios (HR) and $95 \%$ confidence interval (CI) for the relationship between node status and 5-year DFS or 5-year BCSS within each

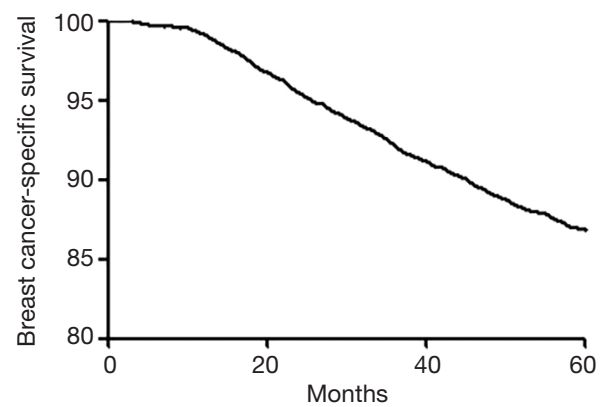

Figure 2 Kaplan-Meier curves for 5-year BCSS. BCSS, breast cancer-specific survival.

molecular subtype. The DFS was defined by the date of primary diagnosis to any case of recurrence (localregional, contralateral breast or distant) or death. We calculated BCSS from the time of diagnose to death from breast cancer. All statistical tests were two-sided, and $P$ values $<0.05$ were considered statistically significant.

\section{Results}

\section{Clinicopatbological characteristics}

A total of 2,089 eligible patients with breast cancer were included in the study. The median follow-up time was 6.4 years. Patient's demographics and clinicopathological characteristics are listed in Table 1. Using the TNM staging system, tumors were categorized based on the number of invasive lymph nodes in N0 ( $\mathrm{n}=1,119,53.6 \%), \mathrm{N} 1(\mathrm{n}=521$, $25.0 \%), \mathrm{N} 2(\mathrm{n}=256,12.2 \%)$, and N3 ( $\mathrm{n}=193,9.2 \%)$. The percentage of molecular subtypes among the patients in the study was as follows; luminal A in $22.2 \%$, luminal B in $31.3 \%$, luminal HER2 in $14.1 \%$, HER2 in $16.3 \%$, and TNBC in $16.1 \%$.

\section{Survival analysis (5-year DFS and 5-year BCSS)}

In the entire group the 5 -year DFS and BCSS rate were $1,631 / 78.1 \%$ and $1,814 / 86.8 \%$ (Figures 1,2 ). Using univariate Cox regression, tumor size, node status, histologic grade, Ki-67, and molecular subtype were significant prognostic factors for 5-year DFS and BCSS. Age was only significant for 5 -year DFS but not for 5-year BCSS (Tables 2,3). After adjusting for other prognostic factors, the nodal involvement $(\mathrm{N} 1, \mathrm{~N} 2, \mathrm{~N} 3)$ was associated with worse 5-year DFS and 5-year BCSS compared with the node negative group. 
Table 2 Univariate and multivariate analyses of DFS

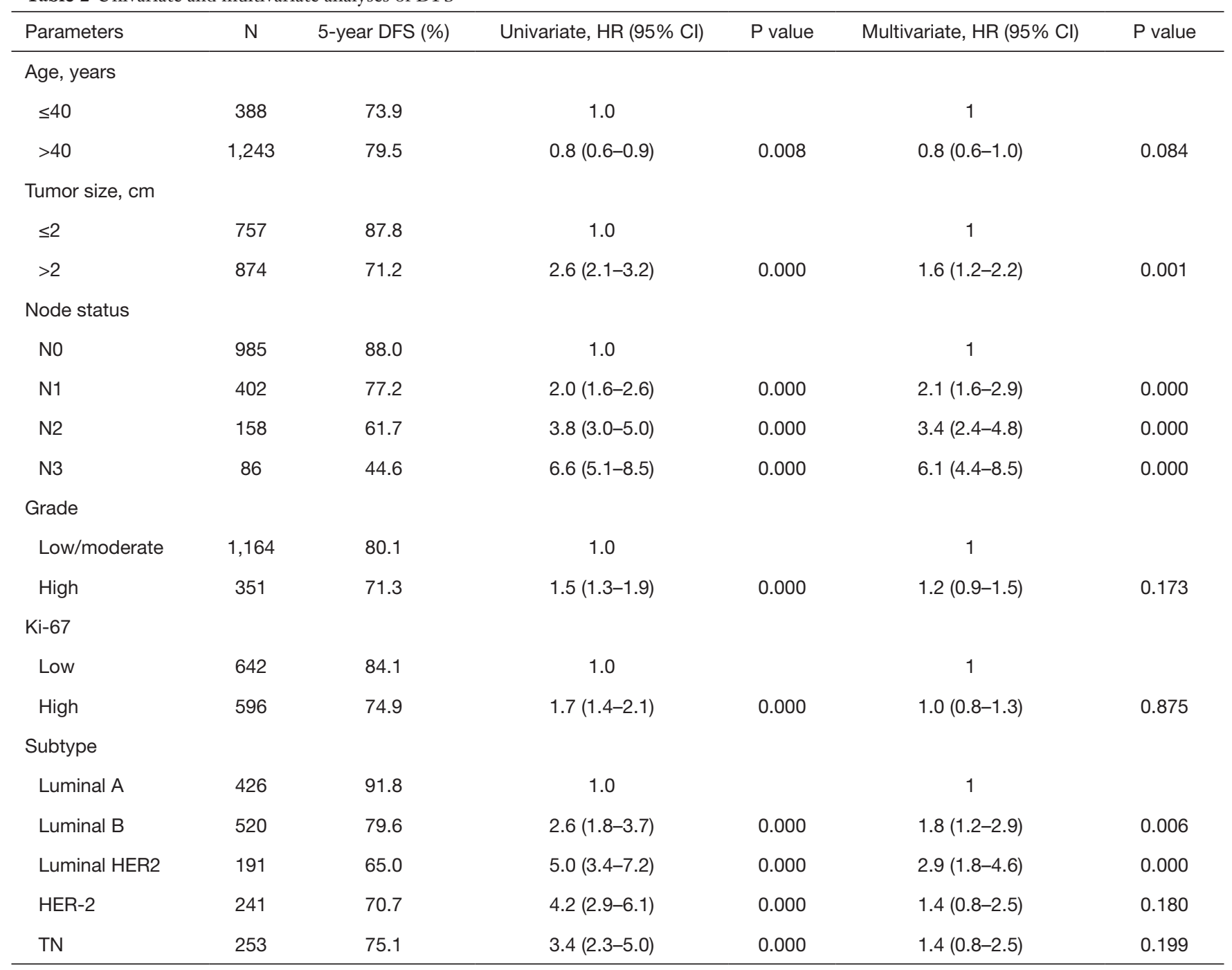

HR, hazard ratios; DFS, disease-free survival.

\section{Stratification by molecular subtype}

In Table 4 we show that the luminal A and TN subtypes predict a lower incidence of nodal involvement (39.9\% and $40.9 \%$ ) compared with luminal HER2 and luminal B subtypes (55.1\% and 52.4\%). Similarly, the luminal B, luminal HER2, and HER2 subtypes present a greater percentage of high-volume nodal involvement ( $\geq 4$ positive LN) compared with luminal A disease. Multivariable Cox proportional hazards regression was used to describe the association between node status and 5-year DFS or 5-year BCSS in different constructed molecular subtypes as shown in Table 5. After adjusting for age, tumor size, and histological grade there was no difference in 5 -year DFS and 5 -year BCSS between the N1 or N2 and N0 groups of patients with luminal A disease. For women with the HER2 subtype, similarly, there was no difference in 5 -year DFS and 5-year BCSS between the N1 and N0 groups. However, nodal involvement (N1, N2, and N3) patients showed a significant difference for 5-year DFS and 5-year BCSS compared to the reference group (N0) with the luminal B, luminal HER2, and TN subtypes.

\section{Discussion}

Several studies have reported that nodal involvement may be an independent prognostic parameter which is associated 
Table 3 Univariate and multivariate analyses of BCSS

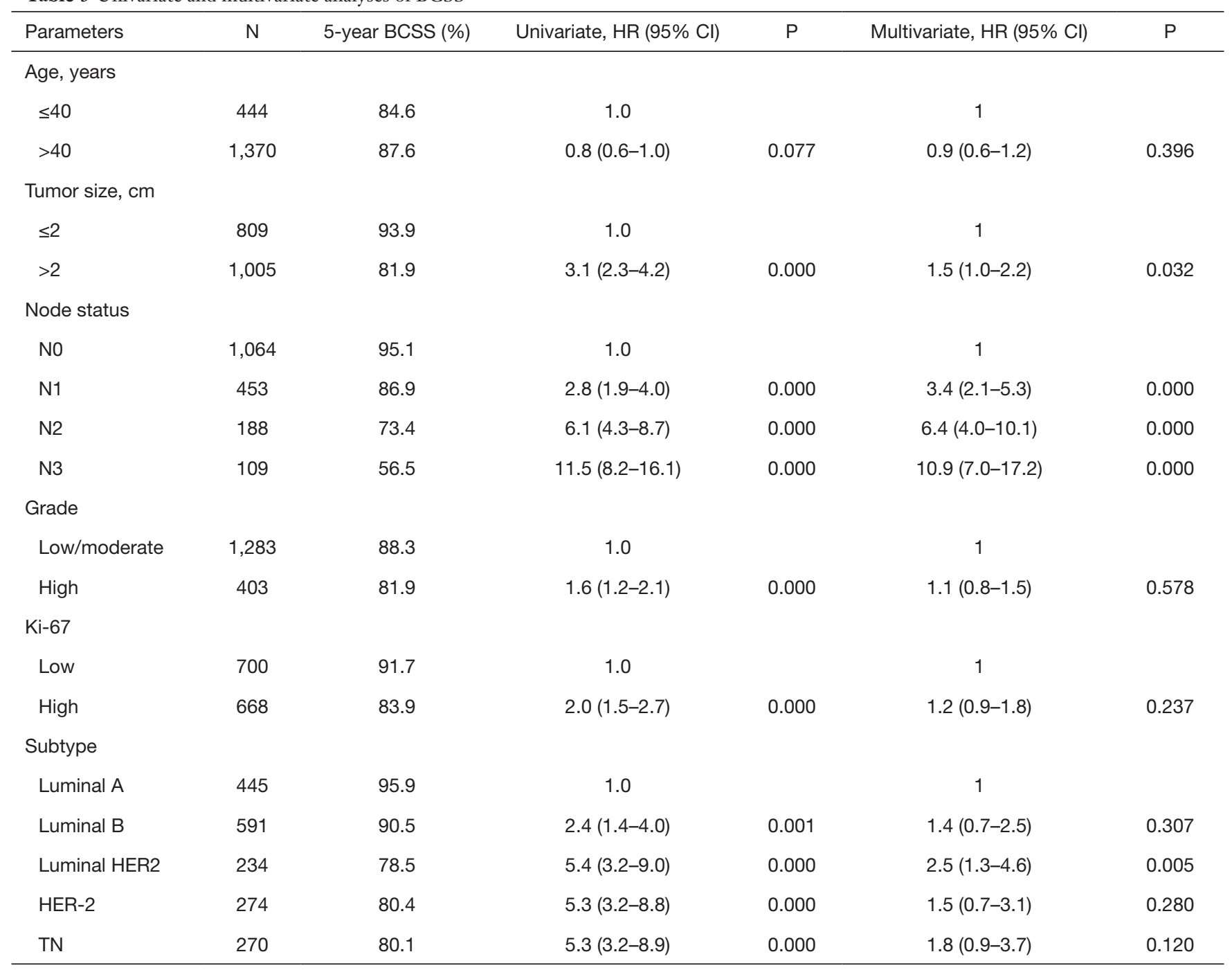

Table 4 Correlation of molecular subtypes and the number of nodal involvements

\begin{tabular}{lcc}
\hline Subtype & Any nodal involvement $(\geq 1$ positive LN), \% & High-volume nodal involvement $(\geq 4$ positive LN), \% \\
\hline Luminal A & 39.90 & 12.50 \\
Luminal B & 52.40 & 25.60 \\
Luminal HER2 & 55.10 & 26.20 \\
HER-2 & 41.90 & 25.50 \\
TN & 40.90 & 17.80 \\
\hline
\end{tabular}


Table 5 Node status and 5-year DFS and 5-year BCSS by molecular subtype

\begin{tabular}{|c|c|c|c|c|c|c|c|}
\hline Subtype/node & Patients, n (\%) & 5-year DFS, n (\%) & $\operatorname{HR}(95 \% \mathrm{Cl})^{*}$ & $\mathrm{P}$ & 5-year BCSS, n (\%) & $\mathrm{HR}(95 \% \mathrm{Cl})^{*}$ & $\mathrm{P}$ \\
\hline No & $279(60.1)$ & $264(94.6)$ & 1 & & $270(96.8)$ & 1 & \\
\hline N1 & $127(27.4)$ & $115(90.6)$ & $1.5(0.7-3.4)$ & 0.260 & $121(95.3)$ & $1.7(0.5-5.1)$ & 0.365 \\
\hline N2 & $41(8.8)$ & $36(87.8)$ & $2.2(0.7-6.3)$ & 0.162 & $40(97.6)$ & $0.8(0.1-7.1)$ & 0.872 \\
\hline Luminal B & 653 & $520(79.6)$ & & & $591(90.5)$ & & \\
\hline NO & 311 (47.6) & 277 (89.1) & 1 & & $303(97.4)$ & 1 & \\
\hline $\mathrm{N} 1$ & $175(26.8)$ & $141(80.6)$ & $1.7(1.0-2.8)$ & 0.050 & $161(92.0)$ & $2.5(1.0-6.3)$ & 0.043 \\
\hline N2 & $98(15.0)$ & $65(66.3)$ & $2.8(1.7-4.8)$ & 0.000 & 79 (80.6) & $6.1(2.5-14.6)$ & 0.000 \\
\hline No & $132(44.9)$ & $104(78.8)$ & 1 & & $122(92.4)$ & 1 & \\
\hline $\mathrm{N} 1$ & 85 (28.9) & $50(58.8)$ & $2.0(1.2-3.4)$ & 0.009 & $67(78.8)$ & $3.4(1.5-7.7)$ & 0.003 \\
\hline N2 & $44(15.0)$ & $22(50.0)$ & $2.7(1.5-5.0)$ & 0.001 & $27(61.4)$ & $5.3(2.2-12.4)$ & 0.000 \\
\hline N3 & $33(11.2)$ & $15(45.4)$ & $2.5(1.3-4.8)$ & 0.005 & $18(54.5)$ & $5.4(2.2-12.8)$ & 0.000 \\
\hline HER2 & 341 & $241(70.7)$ & & & $274(80.4)$ & & \\
\hline No & $198(58.1)$ & $166(83.8)$ & 1 & & $185(93.4)$ & 1 & \\
\hline N1 & $56(16.4)$ & $45(80.4)$ & 1.1 & 0.774 & $50(89.3)$ & $1.7(0.6-4.5)$ & 0.292 \\
\hline $\mathrm{N} 2$ & $39(11.4)$ & $16(41.0)$ & 4.5 & 0.000 & $21(53.8)$ & $8.3(4.0-17.2)$ & 0.000 \\
\hline N3 & $26(7.7)$ & 9 (34.6) & $6.9(3.6-13.5)$ & 0.000 & $11(42.3)$ & $10.0(4.6-21.6)$ & 0.000 \\
\hline
\end{tabular}

*, adjusted for age, tumor size, histological grade.

with a poor prognosis (19-21). However, there are limited published studies on this topic that evaluate the impact of node status on prognosis by molecular subtype in women with early breast cancer. Hence, we used a large cohort of cases obtained from 2,089 women with breast cancer to conduct a retrospective study. We found that nodal involvement is an independent predictors of the 5 -year DFS or 5 -year BCSS in patients with breast cancer. Even when adjustments were made for patient age, tumor size, grade, $\mathrm{Ki}-67$, and subtypes, a multivariate analysis showed that lymph node involvement implies a worse 5 -year DFS and 5 -year BCSS, which is consistent with the report by Ataseven et al. (19). Similarly, Liao et al. reported that a higher positive number of lymph nodes was associated with an inferior 5-year OS (13). On the face of it, nodal involvement and a higher number of positive nodes does increase the risk of survival $(20,21)$.

Recent studies have suggested that some molecular subtypes are associated with the node status and an increased risk of nodal involvement (22-25). Our study indicates that the luminal A and TN subtypes predict a lower incidence of nodal involvement. However, a greater percentage of 
nodal involvement and high-volume nodal involvement ( $\geq 4$ positive $\mathrm{LN}$ ) was found for women with the luminal B, luminal HER2, and HER2 subtypes. Wiechmann et al. have reported that after controlling for patient age, tumor size, lymphovascular invasion, and grade, that the luminal B and luminal HER2 subtype tumors were more likely to manifest nodal involvement or more metastatic lymph nodes compared with patients with luminal A (26). Our data are also consistent with a previously reported study by Crabb et al. that indicates that the TNBC is associated with a lower incidence of nodal involvement than other subtypes despite its poor prognosis (23). Similar results had been reported by studies from Howland et al. and Bhargava et al. $(22,27)$. Our study supports the reports that luminal B, luminal HER2, and HER2 subtypes are associated with a higher likelihood of nodal involvement and high-volume (four or more positive lymph nodes) axillary metastasis. Furthermore, we found that the node status is associated with molecular subtype and has the prognostic value for predicting 5-year DFS and BCSS in molecular subtypes. Luminal B, luminal HER2 and TN subtypes, are more aggressive subtypes than luminal A and always considered to be associated with an unfavorable prognosis at the diagnosis of breast cancer. For the patients with these subtypes, nodal involvement had a statistically significant association with a worse 5-year DFS and 5-year BCSS compared with the N0 group. Similarly, Liao et al. had reported a large study with 1,399 patients to investigate the association between node status and prognosis of breast cancer by molecular subtype. Their results indicated that there were significant differences in 5-year RFS and OS according to the node status among the luminal B and TN subtypes. After adjusting for age and tumor size for OS and RFS by multivariate Cox proportional hazard analysis, a statistically significant difference remained among the luminal B and luminal HER2 subtypes (13). Among women with luminal B, luminal HER2 and TN subtypes, nodal involvement seems to remain an independent prognostic factor.

In women with luminal A subtype, our report indicated that the N1 or N2 group with luminal A disease did not have a worse 5-year DFS and 5-year BCSS compared with the N0 group. The luminal A subtype is characterized by the expression of hormone receptors and potential sensitivity to endocrine therapy. Some studies have reported than luminal A disease with nodal involvement may benefit more from adjuvant endocrine therapy or the effect of chemotherapy than do node negative patients. We hypothesize that the N1 or N2 group with a luminal A subtype benefits more from adjuvant endocrine therapy or the effect of chemotherapy than the N0 group and these treatments may help to reduce outcome disparities associated with node status among those with higher-risk luminal A tumors in particular. Nodal involvement seems not to be an independent risk factor among luminal A tumors.

For women with HER2 breast cancer, our data indicates that there was no clear decreased risk of BCSS and DFS for the N1 group compared with the N0 group when controlling for other conventional prognostic factors. Several large clinical trials have reported that 1 year of treatment with trastuzumab after adjuvant chemotherapy significantly improves DFS or overall survival among women with node-positive and high-risk node-negative HER2 positive breast cancer $(28,29)$. A randomized controlled trial reported that women with lymph node involvement benefit more from trastuzumab compared with node negative patients (30). These studies suggested that the N1 group with HER2 subtype benefit more from trastuzumab than the N0 group, and trastuzumab treatment may help to cancel out the survival difference from different degrees of lymph node involvement. Furthermore, there was a certain proportion of the N0 group who were HER2 positive who cannot accept trastuzumab for economic reasons. So, it seems that the N0 with HER2 patients who were not treated with trastuzumab had a similar prognosis to the N1 group; despite not receiving trastuzumab they did not do worse. Theoretically, nodal involvement could be an independent prognostic factor among the HER2 subtype if more N0 patients accepted treatment with trastuzumab.

Our results support that the relationship between node status and DFS or BCSS varies with molecular subtype. After controlling for other prognostic factors, we observed that there was no significant difference in survival with luminal A subtype between patients with the involvement of less than 10 lymph node and lymph node negative patients, although nodal involvement seem to be an independent predictor of a worse prognosis for patients with luminal B, luminal HER2, and triple negative subtypes. Certainly, there are several limitations to our study. First, this is a retrospective single-institution study and all patients enrolled were Chinese women and results may not apply to other ethnic population with breast cancer. The second limitation of this study was an insufficient study population and follow-up. Furthermore, the information about adjuvant therapy was lacking. However, to the best of our knowledge, this study is one of the largest similar studies reported to date. 


\section{Conclusions}

The prognostic significance of node status varies with molecular subtype. Nodal involvement seems to be associated with worse survival in women with the luminal B, luminal HER2, and triple-negative subtypes, but not in the luminal A disease.

\section{Acknowledgments}

Funding: This work was supported by grants from the Science and Technology Programme of Quanzhou (2018N073S).

\section{Footnote}

Reporting Checklist: The authors have completed the STROBE reporting checklist. Available at http://dx.doi. org/10.21037/tcr-20-1117

Data Sharing Statement: Available at http://dx.doi. org/10.21037/tcr-20-1117

Peer Review File: Available at http://dx.doi.org/10.21037/tcr20-1117

Conflicts of Interest: All authors have completed the ICMJE uniform disclosure form (available at http://dx.doi. org/10.21037/tcr-20-1117). The authors have no conflicts of interest to declare.

Ethical Statement: The authors are accountable for all aspects of the work in ensuring that questions related to the accuracy or integrity of any part of the work are appropriately investigated and resolved. All methods were carried out in accordance with relevant guidelines and regulations. The study was conducted in accordance with the Declaration of Helsinki (as revised in 2013). The study was approved by Affiliated Union Hospital of Fujian Medical University Ethics committee (No. 002934) and informed consent was taken from all the patients.

Open Access Statement: This is an Open Access article distributed in accordance with the Creative Commons Attribution-NonCommercial-NoDerivs 4.0 International License (CC BY-NC-ND 4.0), which permits the noncommercial replication and distribution of the article with the strict proviso that no changes or edits are made and the original work is properly cited (including links to both the formal publication through the relevant DOI and the license). See: https://creativecommons.org/licenses/by-nc-nd/4.0/.

\section{References}

1. Siegel R, Naishadham D, Jemal A Cancer statistics, 2012. CA Cancer J Clin 2012;62:10-29.

2. Ferlay J, Soerjomataram I, Dikshit R, et al. Cancer incidence and mortality worldwide: sources, methods and major patterns in GLOBOCAN 2012. Int J Cancer 2015;136:E359-86.

3. Fitzmaurice C, Dicker D, Pain A, et al. The Global Burden of Cancer 2013. JAMA Oncol 2015;1:505-27.

4. Vinh-Hung V, Burzykowski T, Cserni G, et al. Functional form of the effect of the numbers of axillary nodes on survival in early breast cancer. Int J Oncol 2003;22:697-704.

5. Jatoi I, Hilsenbeck SG, Clark GM, et al. Significance of axillary lymph node metastasis in primary breast cancer. J Clin Oncol 1999;17:2334-40.

6. Perou CM, Sorlie T, Eisen MB, et al. Molecular portraits of human breast tumours. Nature 2000;406:747-52.

7. Rakha EA, Reis-Filho JS, Baehner F, et al. Breast cancer prognostic classification in the molecular era: the role of histological grade. Breast Cancer Res 2010;12:207.

8. Voduc KD, Cheang MC, Tyldesley S, et al. Breast cancer subtypes and the risk of local and regional relapse. J Clin Oncol 2010;28:1684-91.

9. Engstrom MJ, Opdahl S, Hagen AI, et al. Molecular subtypes, histopathological grade and survival in a historic cohort of breast cancer patients. Breast Cancer Res Treat 2013;140:463-73.

10. Rugină VG, Mihalcea D, Pricop F. The lymph nodes status--prognostic factor in breast cancer. Rev Med Chir Soc Med Nat Iasi 2011;115:839-44.

11. Salhia B, Tapia C, Ishak EA, et al. Molecular subtype analysis determines the association of advanced breast cancer in Egypt with favorable biology. Bmc Womens Health 2011;11:44.

12. He ZY, Wu SG, Yang Q, et al. Breast Cancer Subtype is Associated With Axillary Lymph Node Metastasis: A Retrospective Cohort Study. Medicine (Baltimore) 2015;94:e2213.

13. Liao GS, Chou YC, Hsu HM, et al. The prognostic value of lymph node status among breast cancer subtypes. Am J Surg 2015;209:717-24.

14. Lian W, Fu F, Lin Y, et al. The Impact of Young Age for 
Prognosis by Subtype in Women with Early Breast Cancer. Sci Rep 2017;7:11625.

15. Cheang MC, Chia SK, Voduc D, et al. Ki-67 index, HER2 status, and prognosis of patients with luminal B breast cancer. J Natl Cancer Inst 2009;101:736-50.

16. Desmedt C, Haibe-Kains B, Wirapati P, et al. Biological processes associated with breast cancer clinical outcome depend on the molecular subtypes. Clin Cancer Res 2008;14:5158-65.

17. Coates AS, Winer EP, Goldhirsch A, et al. Tailoring therapies--improving the management of early breast cancer: St Gallen International Expert Consensus on the Primary Therapy of Early Breast Cancer 2015. Ann Oncol 2015;26:1533-46.

18. Goldhirsch A, Winer EP, Coates AS, et al. Personalizing the treatment of women with early breast cancer: highlights of the St Gallen International Expert Consensus on the Primary Therapy of Early Breast Cancer 2013. Ann Oncol 2013;24:2206-23.

19. Ataseven B, Kummel S, Weikel W, et al. Additional prognostic value of lymph node ratio over $\mathrm{pN}$ staging in different breast cancer subtypes based on the results of 1,656 patients. Arch Gynecol Obstet 2015;291:1153-66.

20. Bai LS, Chen C, Gong YP, et al. Lymph node ratio is more predictive than traditional lymph node stratification in lymph node positive invasive breast cancer. Asian Pac J Cancer Prev 2013;14:753-57.

21. Jones T, Neboori H, Wu H, et al. Are breast cancer subtypes prognostic for nodal involvement and associated with clinicopathologic features at presentation in earlystage breast cancer? Ann Surg Oncol 2013;20:2866-72.

22. Howland NK, Driver TD, Sedrak MP, et al. Lymph node involvement in immunohistochemistry-based molecular classifications of breast cancer. J Surg Res 2013;185:697-703.

23. Crabb SJ, Cheang MC, Leung S, et al. Basal breast cancer molecular subtype predicts for lower incidence of axillary lymph node metastases in primary breast cancer. Clin Breast Cancer 2008;8:249-56.

24. Brouckaert O, Pintens S, Van Belle V, et al. Short-term outcome of primary operated early breast cancer by hormone and HER2 receptors. Breast Cancer Res Treat 2009;115:349-58.

25. Van Calster B, Vanden BI, Drijkoningen M, et al. Axillary lymph node status of operable breast cancers by combined steroid receptor and HER2 status: triple positive tumours are more likely lymph node positive. Breast Cancer Res Treat 2009;113:181-87.

26. Wiechmann L, Sampson M, Stempel M, et al. Presenting features of breast cancer differ by molecular subtype. Ann Surg Oncol 2009;16:2705-10.

27. Bhargava R, Striebel J, Beriwal S, et al. Prevalence, morphologic features and proliferation indices of breast carcinoma molecular classes using immunohistochemical surrogate markers. Int J Clin Exp Pathol 2009;2:444-55.

28. Perez EA, Romond EH, Suman VJ, et al. Trastuzumab plus adjuvant chemotherapy for human epidermal growth factor receptor 2-positive breast cancer: planned joint analysis of overall survival from NSABP B-31 and NCCTG N9831. J Clin Oncol 2014;32:3744-52.

29. Perez EA, Romond EH, Suman VJ, et al. Four-year follow-up of trastuzumab plus adjuvant chemotherapy for operable human epidermal growth factor receptor 2-positive breast cancer: joint analysis of data from NCCTG N9831 and NSABP B-31. J Clin Oncol 2011;29:3366-73.

30. Cameron D, Piccart-Gebhart MJ, Gelber RD, et al. 11 years' follow-up of trastuzumab after adjuvant chemotherapy in HER2-positive early breast cancer: final analysis of the HERceptin Adjuvant (HERA) trial. Lancet 2017;389:1195-205.
Cite this article as: Lian W, Fu F, Chen D, Wang C. Effect of node status on breast cancer survival by subtype: a single-center retrospective cohort study. Transl Cancer Res 2020;9(10):5900-5908. doi: $10.21037 /$ tcr-20-1117 